\title{
Microdosimetry for Targeted Alpha Therapy of Cancer
}

\author{
Chen-Yu Huang, ${ }^{1}$ Susanna Guatelli, ${ }^{2}$ Bradley M. Oborn, ${ }^{2,3}$ and Barry J. Allen ${ }^{4}$ \\ ${ }^{1}$ Centre for Experimental Radiation Oncology, St. George Clinical School, University of New South Wales, \\ Kogarah, NSW 2217, Australia \\ ${ }^{2}$ Illawarra Cancer Care Centre, Wollongong, NSW 2522, Australia \\ ${ }^{3}$ Centre for Medical Radiation Physics, University of Wollongong, NSW 2522, Australia \\ ${ }^{4}$ Ingham Institute of Applied Medical Research, Faculty of Medicine, University of Western Sydney, Liverpool, NSW 2170, Australia
}

Correspondence should be addressed to Chen-Yu Huang, cyhuangsysu@gmail.com

Received 3 July 2012; Accepted 25 July 2012

Academic Editor: Eva Bezak

Copyright (C) 2012 Chen-Yu Huang et al. This is an open access article distributed under the Creative Commons Attribution License, which permits unrestricted use, distribution, and reproduction in any medium, provided the original work is properly cited.

Targeted alpha therapy (TAT) has the advantage of delivering therapeutic doses to individual cancer cells while reducing the dose to normal tissues. TAT applications relate to hematologic malignancies and now extend to solid tumors. Results from several clinical trials have shown efficacy with limited toxicity. However, the dosimetry for the labeled alpha particle is challenging because of the heterogeneous antigen expression among cancer cells and the nature of short-range, high-LET alpha radiation. This paper demonstrates that it is inappropriate to investigate the therapeutic efficacy of TAT by macrodosimetry. The objective of this work is to review the microdosimetry of TAT as a function of the cell geometry, source-target configuration, cell sensitivity, and biological factors. A detailed knowledge of each of these parameters is required for accurate microdosimetric calculations.

\section{Introduction}

Targeted alpha therapy (TAT) can provide selective systemic radiotherapy to primary and metastatic tumors (even at a low dose rate and hypoxia region) [1]. It permits sensitive discrimination between target and normal tissue, resulting in fewer toxic side effects than most conventional chemotherapeutic drugs. Monoclonal antibodies (MAbs) that recognize tumor-associated antigens are conjugated to potent alpha emitting radionuclides to form the alpha-immunoconjugate (AIC) (Figure 1). The AIC can be administered by intralesional, orthotopic, or systemic injection. Targeted cancer cells are killed by the short-range alpha radiation, while sparing distant normal tissue cells, giving the minimal toxicity to normal tissue [2].

An alpha particle with energy of 4 to $9 \mathrm{MeV}$ can deposit about $100 \mathrm{keV} / \mu \mathrm{m}$ within a few cell diameters $(40-90 \mu \mathrm{m})$, causing direct DNA double-strand breaks, which lead to cancer cell apoptosis [3]. Cell survival is relatively insensitive to the cell cycle or oxygen status for alpha radiation [4]. TAT is potent enough to eradicate disseminated cancer cells or cancer stem cells that are minimally susceptible to chemo- or radio-resistance. The relative biological effect ( $\mathrm{RBE})$ of alpha particles is from 3 to 7 [5], which means that for the same absorbed dose, the acute biological effects of alpha particles are 3 to 7 times greater than the damage caused by external beam photons or beta radiation.

TAT is ideally suited to liquid cancers or micrometastases [6]. However the regression of metastatic melanoma lesions after systemic TAT in a phase I clinical trial for metastatic melanoma has broadened the application to solid tumors [7]. The observed tumor regression could not be ascribed to killing of all cancer cells in the tumors by TAT and led to the hypothesis that tumors could be regressed by a mechanism called tumor antivascular alpha therapy (TAVAT) [8]. Therapeutic efficacy relates to the extravasation of the AIC through porous tumor vascular walls and widened endothelial junctions into the perivascular space in the solid tumor. The AICs bind to antigenic sites on the membranes of pericytes and contiguous cancer cells around the capillary. The alpha-particle emitters are localized close to the vascular endothelial cells (ECs), which are irradiated by alpha particles and killed. Subsequent tumor capillary closure, causing 


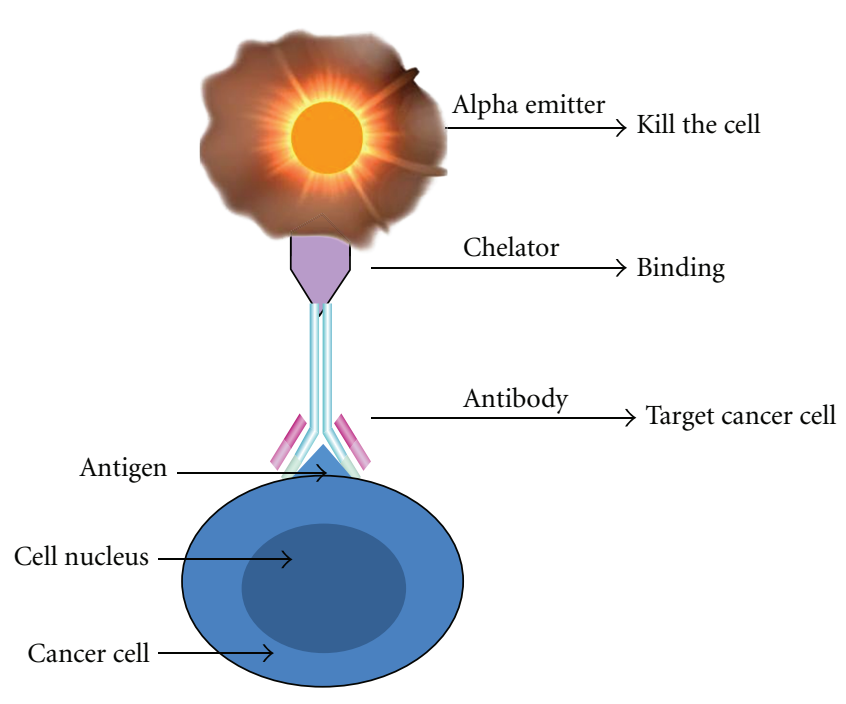

FIgURE 1: Schematic diagram of an AIC targeting a cell.

depletion of oxygen and nutrition, is the likely cause of cancer cell death and tumor regression $[8,9]$.

\section{Microdosimetry}

2.1. Microdosimetry Concept. Radiation dosimetry is the study of the physical properties of radiation energy deposition in tissue. It can be used to optimize treatments and allow comparison of different therapeutic approaches, as well as to study the basic methods of irradiation of biological matter [10]. Radiation dose in conventional external radiotherapy is a macroscopic concept. Upon the properties of the short path length alpha emissions and the spatial distribution of the radionuclide relative to the small target volumes, microdosimetry is indispensible for TAT to investigate the physical properties of radiation energy deposition in biological cells [11].

The dosimetry of TAT is distinguished from that of beta immunotherapy [12] or external beam radiotherapy in three different ways.

(1) Short path length of alpha particles. The high energy of alpha particles is deposited in a short range [13]. Some cell nuclei receive multiple alpha particle hits, while others receive no hits. The amounts of energy deposited vary greatly from target to target, leading to a wide frequency distribution [14].

(2) Small target volume. The alpha track length is comparable to cellular/subcellular sizes causing high LET within the small target volume. It is important to understand the differing biological effects on individual cells [15]. Given the energy delivered along an alpha-particle track and its potential cytotoxicity, the dosimetry for estimating mean absorbed dose may not always yield physically or biologically meaningful information of radiation energy deposition in biological cells. Instead, stochastic or microdosimetric methodologies may be required [16].
(3) Nonuniform distribution of radioisotopes. The heterogeneous antigen expression and tumor uptake leads to variable spatial microdosimetric distributions of the AIC [17]. Spatial and temporal changes of the source activity in the target can also occur [4]. When the distribution of radio-labeled antibody is nonuniform, techniques of dose averaging over volumes greater in size than the individual target volumes can become inadequate predictors of the biological effect [18].

The specific energy is the most important quantity for microdosimetry as it can be used to calculate the cancer cell survival rate. Specific energy ( $z \mathrm{~Gy}$ ) is the ratio of the energy deposited ( $\varepsilon$ Joule) to the mass of the target $(m \mathrm{~kg})(1)$ and has the same units as absorbed dose [19]. The mean specific energy equals the absorbed dose [15]. Although microdosimetry is concerned with the same concept of energy deposition per unit mass as dosimetry, the difference in the length of alpha particle and small size of the target volume introduces stochastic effects which are negligible in conventional dosimetry [20].

$$
z=\frac{\varepsilon}{m} \mathrm{~Gy} .
$$

The stochastic quantity of specific energy $z$ can be used to investigate biological effects [21]. The cell survival fraction (SF) is given by

$$
\mathrm{SF}=e^{-z / z_{0}},
$$

where $z_{0}$ is the absorbed dose required to reduce cell survival to $37 \%$ [22].

Many microdosimetric models have been developed since Roesch's initial work [23]. Two different microdosimetric methods can be used. Experimental measurement with high-resolution solid-state microdosimeters is one way. The high spatial resolution and the tissue-equivalence correction detectors have been applied for hadron therapy and Boron neutron capture therapy [24-26]. On the other hand, dose distributions can be calculated with analytical calculations [15] or Monte Carlo techniques based on fundamental physical principles [27]. The latter method is more practical and much less expensive [19].

2.2. Microdosimetry Case Study. It is inappropriate to evaluate the background dose for radioimmunotherapy, especially for TAT by conventional dosimetry. For example, in the phase I clinical trial for metastatic melanoma, patients received up to $25 \mathrm{mCi}$ of ${ }^{213} \mathrm{Bi}$ [7]. Assuming that all the activity injected in patient remains in the blood, $25 \mathrm{mCi}$ corresponds to $3.65 \times 10^{12} 213 \mathrm{Bi}$ atoms in the macrodosimetry point of view. Taking the average energy of $8.32 \mathrm{MeV}$, the total energy lost would be 4.8 Joules. The absorbed dose $(z)$ received by blood ( $5 \mathrm{~L}$ ) would be calculated by

$$
z=\frac{\varepsilon}{m}=\frac{4.8 \mathrm{~J}}{5 \mathrm{~kg}} \approx 1 \mathrm{~Gy} .
$$

The above dosimetry would indicate that the blood system would receive an absorbed dose of $\sim 1$ Gy from alpha particle 
TABLE 1: Experimental values of $z_{0}$ for in vitro exposure to alpha radiation.

\begin{tabular}{lccc}
\hline Cell line & AIC & $z_{0}(\mathrm{~Gy})$ & Reference \\
\hline MCF7 & ${ }^{225}$ Ac-Herceptin & 0.27 & {$[47]$} \\
BT & ${ }^{225}$ Ac-Herceptin & 0.37 & {$[47]$} \\
MDA & ${ }^{225}$ Ac-Herceptin & 0.53 & {$[47]$} \\
Line 1 & ${ }^{213}$ Bi-13A & 1.4 & {$[36]$} \\
EMT-6 & ${ }^{213} \mathrm{Bi}-13 \mathrm{~A}$ & 1.7 & {$[36]$} \\
\hline
\end{tabular}

irradiation. The risk for unwanted radiation exposures of normal tissues would be too high.

However, by using Geant 4 Monte Carlo microdosimetry calculation, the actual dose to endothelial cells is $\sim 2 \mathrm{cGy}$ and to lymphocyte is $\sim 10 \mathrm{cGy}$ [28], being $2 \%$ and $10 \%$ of the macroscopic absorbed dose, respectively, and is too low to post any serious damage. In other words, unless alphas actually hit their target, their energy is lost to the medium and has no effect on normal tissue.

Memorial Sloan Kettering found that the maximum tolerance dose was in excess of $1 \mathrm{mCi} / \mathrm{kg}$ for bone marrow toxicity [29]. In the melanoma clinical trial [4], $25 \mathrm{mCi}$ converts to $\sim 0.3 \mathrm{mCi} / \mathrm{kg}$ and is well below that value. Thus, the clinical trial result is consistent with the Monte Carlo calculations. The entirely different result from two dosimetry methods shows it is essential to use microdosimetry method for TAT.

\section{Factors Affecting TAT Microdosimetry Result}

3.1. The Choice of the Target. Microdosimetry depends on the choice of target, for example, the entire cell, cell nucleus, or DNA. This is because the size of the target will affect both the energy deposition and the mass which determines the specific energy.

As has been substantiated by in vitro and in vivo experiments, the radiosensitive sites are associated with DNA in the cell nucleus. Microdosimetry research target has fluctuated between DNA and cell nucleus. For alpha particles with a range of a few cell diameters, the cell nucleus is an appropriate choice for the target considering the genome is assumed to be randomly distributed throughout the cell nucleus, and its specific location is not well known [20]. However, under the circumstances that if the particle (e.g., Auger electrons) range is a few $\mu \mathrm{m}$ and the source decays within 1 or $2 \mathrm{~nm}$ of the DNA molecule, radiation dose to the cell nucleus may be inadequate to predict radiation toxicity, and determination of the energy deposition to the DNA molecule may be necessary [30].

3.2. Target and Source Configuration. The TAT microdosimetry dose is highly sensitive to experimental factors such as the nucleus size and source distribution, kinetics of the AIC, and subcellular distribution of the radionuclide.

Because of the short range of alpha particles, even small changes in the thickness or diameter of the cell nucleus can influence the dose distribution. Simplified spheroid models with different cell and nucleus radii are used to model cells $[4,15,31]$. Recently some more realistic models with geometric parameters taken from monolayer or suspended cells measurement were built [32-35].

The position of the source relative to the target cell nucleus is another major factor in determining the hit probability, specific energy, and ultimately the efficacy of TAT [36]. For a spherical single-cell model, the specific energy from activity internalized in the cell nucleus, in the cytoplasm, on the cell membrane, or in the medium can differ as much as 150 times [37]. Rapidly internalized antibodies or radioisotopes are superior because of markedly greater intracellular retention and higher probability of hit. However, caution is needed as some animal studies indicate that the retention of ${ }^{111} \mathrm{In}$ and ${ }^{90} \mathrm{Y}$ is prolonged in normal organs such as bone, liver, and kidney as well [38].

3.3. Cell Sensitivity. The $z_{0}$ value is highly sensitive to experimental factors such as the distribution of DNA within the nucleus (i.e., the phase of the cell cycle) and the number and spatial distribution of the alpha particle sources relative to the target cell nucleus [39]. It is also expected that the in vitro cell sensitivity will vary between different cells within a given tumor. Table 1 shows a survey of TAT in vitro experiments, which illustrated that $z_{0}$ values for cancer cell lines exposed to alpha radiation can vary as much as 6 times, indicating that for a given specific energy, the biological responses can also vary 6 times or more (SF in (2)).

3.4. Radioisotopes. The clinical application of TAT is focused on alpha particle emitters of ${ }^{211} \mathrm{At},{ }^{213} \mathrm{Bi},{ }^{223} \mathrm{Ra}$, and ${ }^{225} \mathrm{Ac}$ [40-42]. The physical properties of these radionuclides, including half-life, mean particle energy, maximum particle energy, and average range in tissue, affect the therapeutic result and are listed in Table $2[43,44]$.

For an AIC with a long residence time in a tumor, a radionuclide with a long half-life will deliver more decays than one with a short half-life $\left(t_{1 / 2}\right)$ for the same initial radioactivity [44]. The number of radionuclides $(N)$ to produce activity $(A)$ is

$$
N=\frac{A}{\lambda}=\frac{A}{\left(0.693 / t_{1 / 2}\right)} .
$$

For TAT aimed at destroying all cancer cells in the tumor, deep penetration and uniform distribution of the AIC would be crucial. Thus, the longer half-life radioisotope would be a better choice. However, if the aim is to destroy tumor capillaries, poor AIC diffusion away from the capillaries and shorter half-life would be an advantage [19].

The longer half-life of ${ }^{225} \mathrm{Ac}$ and the 4 alpha particle emissions (Figure 2) gives greater toxicity and can prolong survival in the mouse xenograft models for several cancers [45]. However, the drawback is that the binding energy of the chelation is not strong enough to withstand the alpha particle recoil energy of the Actinium ion (between 100 and $200 \mathrm{keV}$ ). Daughters of ${ }^{225} \mathrm{Ac}$ will lose tumor selectivity and could diffuse away, causing cell damage in normal tissue $[16,46]$. 
TABLE 2: Physical properties of alpha-particle emitters.

\begin{tabular}{lcccccc}
\hline Radionuclide & $Z$ & Half-life & Mean particle energy* $(\mathrm{MeV})$ & Maximum energy $(\mathrm{MeV})$ & Average range $(\mu \mathrm{m})$ & $<\mathrm{LET}>(\mathrm{keV} / \mu \mathrm{m})$ \\
\hline${ }^{211} \mathrm{At}$ & 85 & $7.2 \mathrm{~h}$ & 6.79 & 7.45 & 60 & 84 \\
${ }^{213} \mathrm{Bi}$ & 83 & $45.6 \mathrm{~min}$ & 8.32 & 8.38 & 4.59 & 45 \\
${ }^{223} \mathrm{Ra}$ & 88 & $11.43 \mathrm{~d}$ & 5.64 & 8.38 & 61 & 81 \\
${ }^{225} \mathrm{Ac}$ & 89 & $10.0 \mathrm{~d}$ & 6.83 & 71 \\
\hline
\end{tabular}

${ }^{*}$ weighted average of emissions.

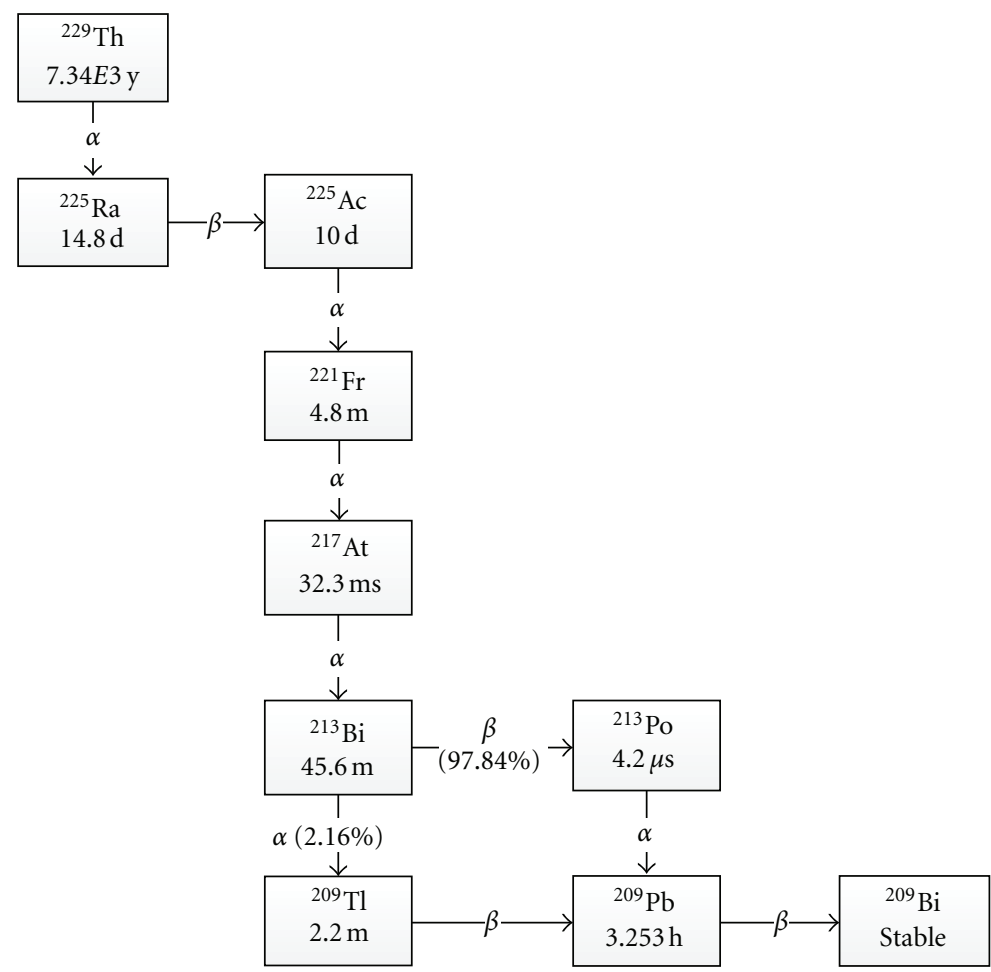

Figure 2: The decay chain of ${ }^{225} \mathrm{Ac}$ and ${ }^{213} \mathrm{Bi}$.

3.5. Biological Factors. There are great complexities of the mammalian cell, the nucleus and its internal structures and pathways, types of DNA damage, and cellular repair. For cancer-cell cluster or solid-tumor modeling, a precise kinetic description of AICs diffusing through cells and saturating antigenic sites is needed for microdosimetry. The number of AICs in the solid tumor depends critically on the capillary permeability and the number of antigens expressed on cells that can vary 10 -fold and more. As such, the calculations rest on realistic assumptions but results, in spite of the quantitative nature of the Monte Carlo calculation, are qualitative only.

\section{Conclusion}

Targeted Alpha Therapy uses radio-isotopes that emit alpha radiation to kill targeted cancer cells. It is most effective in the elimination of single-cancer cells and micrometastases before the tumors grow to become clinically evident. The application has been extended to the treatment of solid tumors by tumor antivascular alpha therapy. Because the short range of alpha particles is comparable to the size of the biological target and the variable distribution of alpha emitters, stochastic processes apply, and Monte Carlo calculations of microdosimetry are indispensible in the investigation of biological response mechanisms.

\section{Acknowledgment}

The authors wish to thank Professor Anatoly Rozenfeld for his support.

\section{References}

[1] M. R. Zalutsky and O. R. Pozzi, "Radioimmunotherapy with $\alpha$-particle emitting radionuclides," Quarterly Journal of Nuclear Medicine and Molecular Imaging, vol. 48, no. 4, pp. 289-296, 2004.

[2] V. Rajkumar, J. L. Dearling, A. Packard, and R. B. Pedley, "Research Spotlight-radioimmunotherapy: optimizing delivery to solid tumors," Therapeutic Delivery, vol. 2, no. 5, pp. 567-572, 2011.

[3] G. Sgouros, "Alpha-particles for targeted therapy," Advanced Drug Delivery Reviews, vol. 60, no. 12, pp. 1402-1406, 2008. 
[4] J. C. Roeske and T. G. Stinchcomb, "Dosimetric framework for therapeutic alpha-particle emitters," Journal of Nuclear Medicine, vol. 38, no. 12, pp. 1923-1929, 1997.

[5] M. R. McDevitt, G. Sgouros, R. D. Finn et al., "Radioimmunotherapy with alpha-emitting nuclides," European Journal of Nuclear Medicine, vol. 25, no. 9, pp. 1341-1351, 1998.

[6] J. G. Jurcic, S. M. Larson, G. Sgouros, M. R. McDevitt, R. D. Finn, and C. R. Divgi, "Targeted $\alpha$ particle immunotherapy for myeloid leukemia," Blood, vol. 100, no. 4, pp. 1233-1239, 2002.

[7] C. Raja, P. Graham, S. M. Abbas Rizvi et al., "Interim analysis of toxicity and response in phase 1 trial of systemic targeted alpha therapy for metastatic melanoma," Cancer Biology and Therapy, vol. 6, no. 6, pp. 846-852, 2007.

[8] B. J. Allen, C. Raja, S. Rizvi, E. Y. Song, and P. Graham, "Tumour anti-vascular alpha therapy: a mechanism for the regression of solid tumours in metastatic cancer," Physics in Medicine and Biology, vol. 52, no. 13, pp. L15-L19, 2007.

[9] B. J. Allen, "Can $\alpha$-radioimmunotherapy increase efficacy for the systemic control of cancer?" Immunotherapy, vol. 3, no. 4, pp. 455-458, 2011.

[10] M. Bardies and P. Pihet, "Dosimetry and microdosimetry of targeted radiotherapy," Current Pharmaceutical Design, vol. 6, no. 14 , pp. 1469-1502, 2000.

[11] R. F. Hobbs, S. Baechler, D. X. Fu et al., "A model of cellular dosimetry for macroscopic tumors in radiopharmaceutical therapy," Medical Physics, vol. 38, no. 6, pp. 2892-2903, 2011.

[12] T. C. Karagiannis, "Comparison of different classes of radionuclides for potential use in radioimmunotherapy," Hellenic Journal of Nuclear Medicine, vol. 10, no. 2, pp. 82-88, 2007.

[13] R. M. Macklis, B. M. Kinsey, A. I. Kassis et al., "Radioimmunotherapy with alpha-particle-emitting immunoconjugates," Science, vol. 240, no. 4855, pp. 1024-1026, 1988.

[14] H. Zaidi and G. Sgouros, Eds., Therapeutic Applications of Monte Carlo Calculations in Nuclear Medicine, vol. 9, vol. 30, Institute of physics, London, UK, 2003.

[15] T. G. Stinchcomb and J. C. Roeske, "Analytic microdosimetry for radioimmunotherapeutic alpha emitters," Medical Physics, vol. 19, no. 6, pp. 1385-1394, 1992.

[16] M. R. McDevitt, G. Sgouros, R. D. Finn et al., "Radioimmunotherapy with alpha-emitting nuclides," European Journal of Nuclear Medicine, vol. 25, no. 9, pp. 1341-1351, 1998.

[17] S. Sofou, "Radionuclide carriers for targeting of cancer," International Journal of Nanomedicine, vol. 3, no. 2, pp. 181-199, 2008.

[18] D. T. Goodhead, "Panel discussion: do non-targeted effects impact the relation between microdosimetry and risk?" Radiation Protection Dosimetry, vol. 143, no. 2-4, pp. 554-556, 2011.

[19] C. Y. Huang, B. M. Oborn, S. Guatelli, and B. J. Allen, "Monte Carlo calculation of the maximum therapeutic gain of tumor antivascular alpha therapy," Medical Physics, vol. 39, no. 3, pp. 1282-1288, 2012.

[20] J. L. Humm, J. C. Roeske, D. R. Fisher, and G. T. Y. Chen, "Microdosimetric concepts in radioimmunotherapy," Medical Physics, vol. 20, no. 2, pp. 535-542, 1993.

[21] D. T. Goodhead, "Energy deposition stochastics and track structure: what about the target?" Radiation Protection Dosimetry, vol. 122, no. 1-4, pp. 3-15, 2006.

[22] D. E. Charlton, A. I. Kassis, and S. J. Adelstein, "A comparison of experimental and calculated survival curves for V79 cells grown as monolayers or in suspension exposed to alpha irradiation from 212Bi distributed in the growth medium,"
Radiation Protection Dosimetry, vol. 52, no. 1-4, pp. 311-315, 1994.

[23] W. C. Roesch, "Microdosimetry of internal sources," Radiation Research, vol. 70, no. 3, pp. 494-510, 1977.

[24] A. B. Rosenfeld, G. I. Kaplan, M. G. Carolan et al., "Simultaneous macro and micro dosimetry with MOSFETs," IEEE Transactions on Nuclear Science, vol. 43, no. 6, pp. 2693-2700, 1996.

[25] P. D. Bradley and A. B. Rosenfeld, "Tissue equivalence correction for silicon microdosimetry detectors in boron neutron capture therapy," Medical Physics, vol. 25, no. 11, pp. 22202225, 1998.

[26] P. D. Bradley, A. B. Rosenfeld, B. Allen, J. Coderre, and J. Capala, "Performance of silicon microdosimetry detectors in boron neutron capture therapy," Radiation Research, vol. 151, no. 3, pp. 235-243, 1999.

[27] S. Incerti, N. Gault, C. Habchi et al., "A comparison of cellular irradiation techniques with alpha particles using the Geant4 Monte Carlo simulation toolkit," Radiation Protection Dosimetry, vol. 122, no. 1-4, pp. 327-329, 2006.

[28] C. Y. Huang, S. Guatelli, B. M. Oborn, and B. J. Allen, "Background dose for systemic targeted Alpha therapy," Progress in Nuclear Science and Technology, vol. 2, pp. 187-190, 2011.

[29] T. L. Rosenblat, M. R. McDevitt, D. A. Mulford et al., "Sequential cytarabine and $\alpha$-particle immunotherapy with bismuth213-lintuzumab (HuM195) for acute myeloid leukemia," Clinical Cancer Research, vol. 16, no. 21, pp. 5303-5311, 2010.

[30] H. Nikjoo, S. Uehara, D. Emfietzoglou, and L. Pinsky, "A database of frequency distributions of energy depositions in small-size targets by electrons and ions," Radiation Protection Dosimetry, vol. 143, no. 2-4, pp. 145-151, 2011.

[31] J. L. Humm and L. M. Chin, "A model of cell inactivation by alpha-particle internal emitters," Radiation Research, vol. 134, no. 2, pp. 143-150, 1993.

[32] S. J. Kennel, R. Boll, M. Stabin, H. M. Schuller, and S. Mirzadeh, "Radioimmunotherapy of micrometastases in lung with vascular targeted 213Bi," British Journal of Cancer, vol. 80, no. 1-2, pp. 175-184, 1999.

[33] D. E. Charlton, "The survival of monolayers of cells growing in clusters irradiated by 211 At appended to the cell surfaces," Radiation Research, vol. 151, no. 6, pp. 750-753, 1999.

[34] Y. Kvinnsland, T. Stokke, and E. Aurlien, "Radioimmunotherapy with alpha-particle emitters: microdosimetry of cell with a heterogeneous antigen expression and with various diameters of cells and nuclei," Radiation Research, vol. 155, no. 2, pp. 288-296, 2001.

[35] S. Incerti, H. Seznec, M. Simon, P. Barberet, C. Habchi, and P. Moretto, "Monte Carlo dosimetry for targeted irradiation of individual cells using a microbeam facility," Radiation Protection Dosimetry, vol. 133, no. 1, pp. 2-11, 2009.

[36] S. J. Kennel, M. Stabin, J. C. Roeske et al., "Radiotoxicity of bismuth-213 bound to membranes of monolayer and spheroid cultures of tumor cells," Radiation Research, vol. 151, no. 3, pp. 244-256, 1999.

[37] N. Chouin, K. Bernardeau, F. Davodeau et al., "Evidence of extranuclear cell sensitivity to alpha-particle radiation using a microdosimetric model-I. Presentation and validation of a microdosimetric model," Radiation Research, vol. 171, no. 6, pp. 657-663, 2009.

[38] Z. Yao, K. Garmestani, K. J. Wong et al., "Comparative cellular catabolism and retention of astatine-, bismuth-, and leadradiolabeled internalizing monoclonal antibody," Journal of Nuclear Medicine, vol. 42, no. 10, pp. 1538-1544, 2001.

[39] G. Sgouros, J. C. Roeske, M. R. McDevitt et al., "MIRD pamphlet No. 22 (Abridged): Radiobiology and dosimetry of 
$\alpha$-particle emitters for targeted radionuclide therapy," Journal of Nuclear Medicine, vol. 51, no. 2, pp. 311-328, 2010.

[40] B. Pohlman, J. Sweetenham, and R. M. Macklis, "Review of clinical radioimmunotherapy," Expert Review of Anticancer Therapy, vol. 6, no. 3, pp. 445-461, 2006.

[41] S. Nilsson, R. H. Larsen, S. D. Fosså et al., "First clinical experience with $\alpha$-emitting radium-223 in the treatment of skeletal metastases," Clinical Cancer Research, vol. 11, no. 12, pp. 44514459, 2005.

[42] B. J. Allen, "Clinical trials of targeted alpha therapy for cancer," Reviews on Recent Clinical Trials, vol. 3, no. 3, pp. 185-191, 2008.

[43] X. Zhu, M. R. Palmer, G. M. Makrigiorgos, and A. I. Kassis, "Solid-tumor radionuclide therapy dosimetry: new paradigms in view of tumor microenvironment and angiogenesis," Medical Physics, vol. 37, no. 6, pp. 2974-2984, 2010.

[44] A. I. Kassis, "Therapeutic radionuclides: biophysical and radiobiologic principles," Seminars in Nuclear Medicine, vol. 38, no. 5, pp. 358-366, 2008.

[45] M. R. McDevitt, D. Ma, L. T. Lai et al., "Tumor therapy with targeted atomic nanogenerators," Science, vol. 294, no. 5546, pp. 1537-1540, 2001.

[46] J. Schwartz, J. S. Jaggi, J. A. O'donoghue et al., "Renal uptake of bismuth-213 and its contribution to kidney radiation dose following administration of actinium-225-labeled antibody," Physics in Medicine and Biology, vol. 56, no. 3, pp. 721-733, 2011.

[47] Å. M. Ballangrud, W. H. Yang, S. Palm et al., "Alpha-particle emitting atomic generator (actinium-225)-labeled trastuzumab (Herceptin) targeting of breast cancer spheroids: efficacy versus HER2/neu expression," Clinical Cancer Research, vol. 10, no. 13, pp. 4489-4497, 2004. 


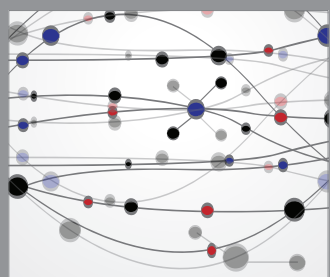

The Scientific World Journal
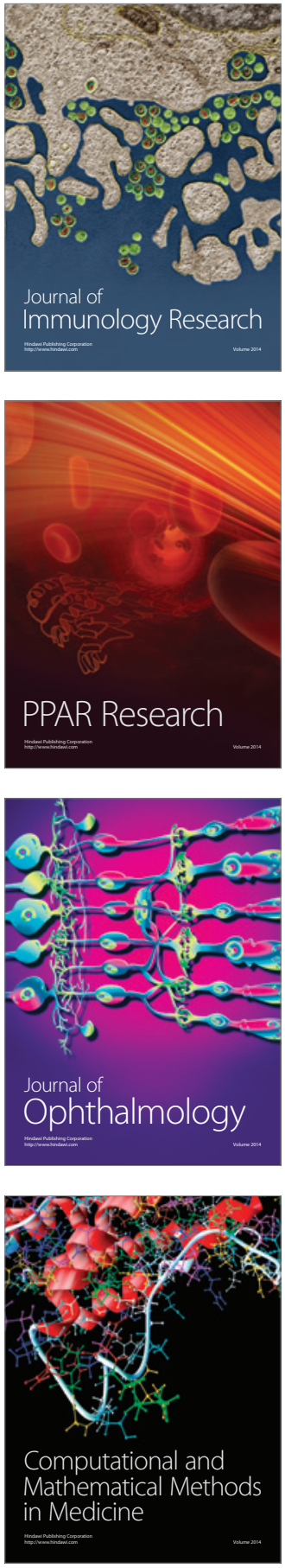

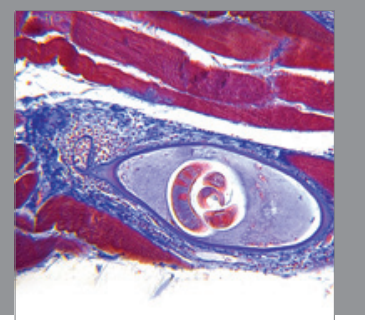

Gastroenterology

Research and Practice
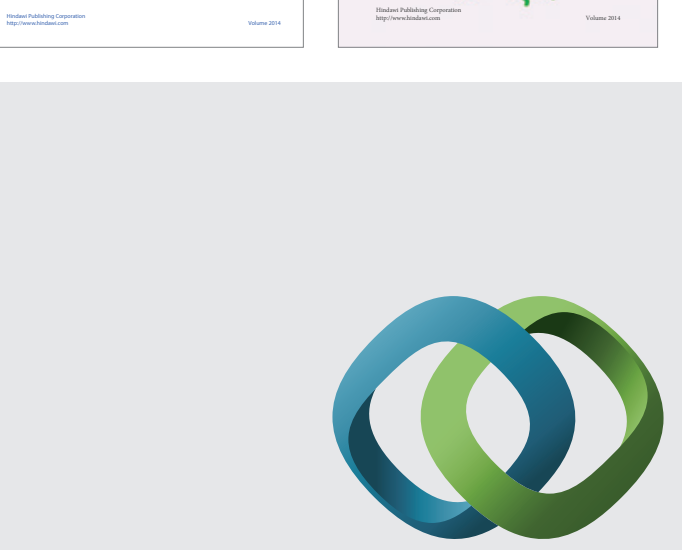

\section{Hindawi}

Submit your manuscripts at

http://www.hindawi.com
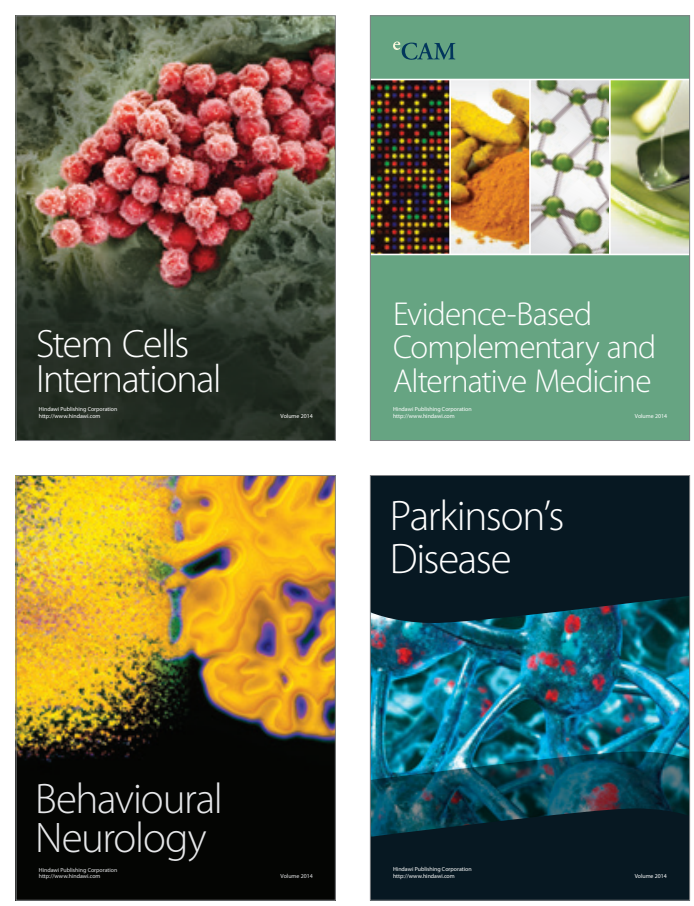

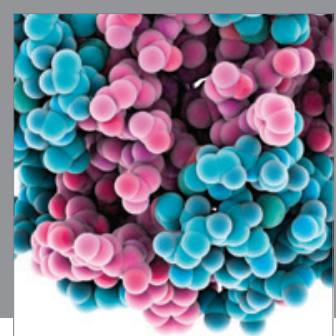

Journal of
Diabetes Research

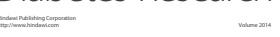

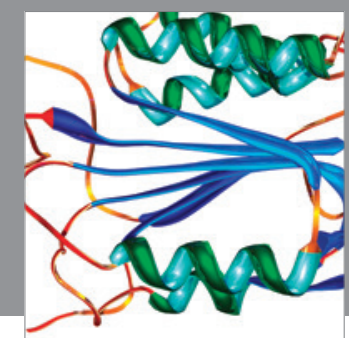

Disease Markers
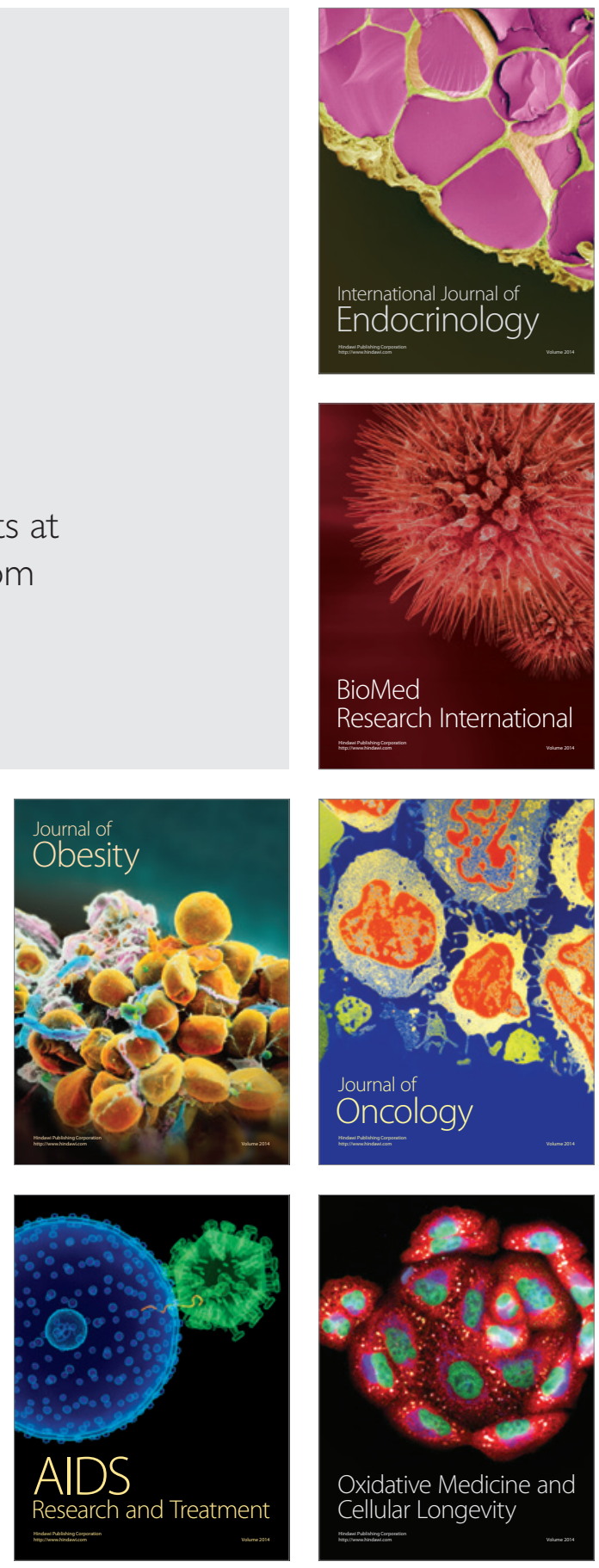